\title{
High-resolution mapping of interstellar clouds with near-infrared scattered light
}

\author{
M. Juvela ${ }^{1}$, V.-M. Pelkonen ${ }^{1}$, P. Padoan ${ }^{2}$ \\ and K. Mattila ${ }^{1}$ \\ ${ }^{1}$ Helsinki University Observatory, FI-00014, Finland \\ ${ }^{2}$ Department of Physics, University of California, San Diego
}

\begin{abstract}
We examine the intensity of scattered near-infrared (NIR) light in the case of interstellar clouds illuminated by the normal interstellar radiation field. We have developed a way to convert the observed surface brightness into estimates of the column density and have estimated the accuracy of the new method. The NIR intensities can be converted into reliable estimates of the column density in regions with $A_{V}$ up to almost 20 magnitudes. The errors can be further reduced with detailed radiative transfer modelling and by using the lower resolution information that is provided by the colour excess data of background stars. Therefore, NIR scattered light is a promising new way to map quiescent interstellar clouds at a high, even sub-arcsecond resolution.
\end{abstract}

Keywords. ISM: clouds, ISM: structure,infrared: ISM, scattering

Current wide-field near-infrared (NIR) instruments make it possible to map the scattered light over large cloud areas (e.g., Foster \& Goodman 2006). Below $A_{V} \sim 10$ magnitudes the surface brightness should be directly proportional to the column density. At higher column densities the scattered intensity shows signs of saturation that starts at the shortest wavelengths. The resulting changes in the intensity ratios between the NIR bands can be used to estimate the level of saturation. We have developed a way to convert observed near-infrared surface brightness values into estimates of column density (Padoan et al. 2006). The reliability of this new method has been examined using simulated observations (Juvela et al. 2006). The studies are based on three-dimensional cloud models obtained from simulations of magnetohydrodynamic turbulence. Maps of near-infrared scattered light are obtained for the model clouds with radiative transfer calculations, and are converted back into column density using the proposed method. We find that NIR intensities can be converted into reliable estimates of the column density in regions with $A_{V}$ up to almost 20 magnitudes. The errors can be further reduced with detailed radiative transfer modelling and, in particular, by using the lower resolution information that is available through the colour excess data of background stars. The NIR scattered light provides a promising new method for the mapping of quiescent interstellar clouds. The main advantage is the high, even sub-arcsecond resolution that can be reached with current instruments. The comparison of surface brightness and extinction data can also serve as a sensitive indicator of changes in dust properties and radiation field within the clouds.

\section{References}

Foster, J. \& Goodman, A. 2006, ApJ 636, L105

Juvela, M., Padoan, P., Pelkonen, V.-M. \& Mattila, K. 2006, A\&A (in press)

Padoan, P., Juvela, M. \& Pelkonen, V.-M. 2006, ApJ 636, L101 